\title{
A Review on Methods of Image Dehazing
}

\author{
Shruti P. Patel \\ PG Student \\ Department of Electronics and Communication \\ Engineering, Uka Tarsadia University, Gujarat.
}

\author{
Manish Nakrani \\ Assistant Professor \\ Department of Electronics And Communication \\ Engineering, UkaTarsadia University, Gujarat.
}

\begin{abstract}
Literature survey is an important for understanding and gaining much more knowledge about the specific area of a subject. The outdoor images captured in inclement weather are degraded due to the presence of haze, fog, rain and so on. Images of scenes captured in bad weather have poor contrasts and colors. This may cause difficulty in detecting the objects in the captured hazy images. Due to haze there is a trouble to many computer vision applications as it diminishes the visibility of the scene. This paper presents a study about different image dehazing methods to remove the haze from the hazy images captured in real world weather conditions to recover a fast and improved quality of haze free images. There is a improvement in terms of contrast, visible range and color fidelity. All these techniques are widely used in many applications such as outdoor Surveillance, object detection, underwater images, etc.
\end{abstract}

\section{General Terms}

Bad weather conditions, Haze, Airlight, Direct attenuation, contrast, color fidelity, Haze model.

\section{Keywords}

Outdoor images, Dehazing, Hazy Images, Transmission map, Polarization, Dark Channel Prior(DCP), Improved DCP (IDCP).

\section{INTRODUCTION}

Images of outdoor scenes are usually degraded by the atmospheric particles like snow, water droplets, etc. So there is a resultant Detroiter in the color and Contrast of the captured image in the bad weather conditions.

\section{What is Haze?}

Haze is an atmospheric phenomenon that obscures the clarity of the sky. All the atmospheric particles are in the range below of $1000 \mathrm{~m}$. Atmospheric particles are fog, moisture, smoke, water droplets, dust, etc

\section{What causes Haze?}

Haze is caused by atmospheric particles suspended in the air. It occurs in many populated areas like industrial areas. Due to haze clarity of images will be degraded.

Haze is a combination of two components Airlight and Direct attenuation.

\section{Haze $=$ Attenuation + Airlight}

While capturing the outdoor image during bad weather condition, the radiance received by the camera from the scene is attenuated along the line of sight. The incoming light is mixed with the light coming from all other directions called the Airlight. It adds whiteness in the image. And the second component Attenuation is the gradual loss in intensity. Due to this the there is significant decay in the color. Amount of scattering depends on the distance between the scene points and the camera. So the degradation is spatially variable.
Dehazing is highly required in consumer photography and computer vision applications. Because many computer vision applications are suffer from low- contrast scene radiance. For example there is a problem of haze in under water images. There are many methods available to remove haze from outdoor image.

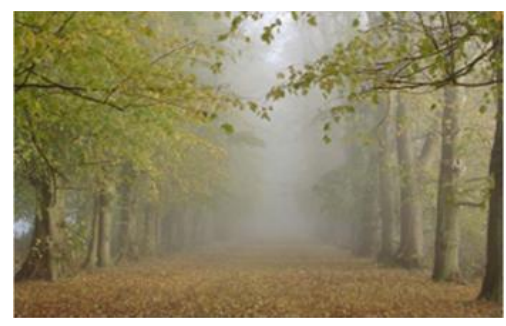

Fig 1. Hazy Image(trees) [16]

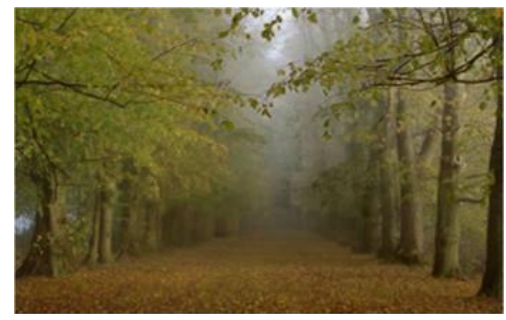

Fig 2. Dehazed Image(trees) [16]

\section{HAZE MODEL}

In surveillance system, remote sensing systems, and underwater, the image appearance is subject to atmospheric conditions such as haze, fog and smoke. Haze formation model is widely used in computer vision and image processing. This model used for the formation of image in the presence of bad atmospheric conditions. Image quality is degraded due to the presence of substantial particles in the atmosphere which have significant size between 1-10 $\mu \mathrm{m}$. The light coming from a camera is absorbed and scattered by these atmospheric particles

Assume that this haze model is linear model. From the definition of linearity in this model only pixel position is changed. Fog is the combination of Airlight and Direct attenuation. This invisibility is occurred by two fundamental phenomenon's: Direct attenuation and Airlight. And it is describe as follow:

$\mathrm{I}(\mathrm{x})=\mathrm{J}(\mathrm{x}) * \mathrm{t}(\mathrm{x})+\mathrm{A} *(1-\mathrm{t}(\mathrm{x})$

Where I (x) is the observed intensity of the xth pixel, $\mathrm{J}(\mathrm{x})$ is the scene radiance vector (the true color that we want to recover), $\mathrm{A}$ is the atmospheric light, and $\mathrm{t}$ is the transmission 
medium describing the portion of the light that is not scattered and reaches direct to the camera.

In the equation first term, $\mathrm{J}(\mathrm{x}) * \mathrm{t}(\mathrm{x})$ is called the direct attenuation; the second term, $\mathrm{A}^{*}(1-\mathrm{t}(\mathrm{x}))$ is called Airlight. This haze model is directly extended to each RGB component of a color image.

\section{DIFFERENT DEHAZING STRATEGIES}

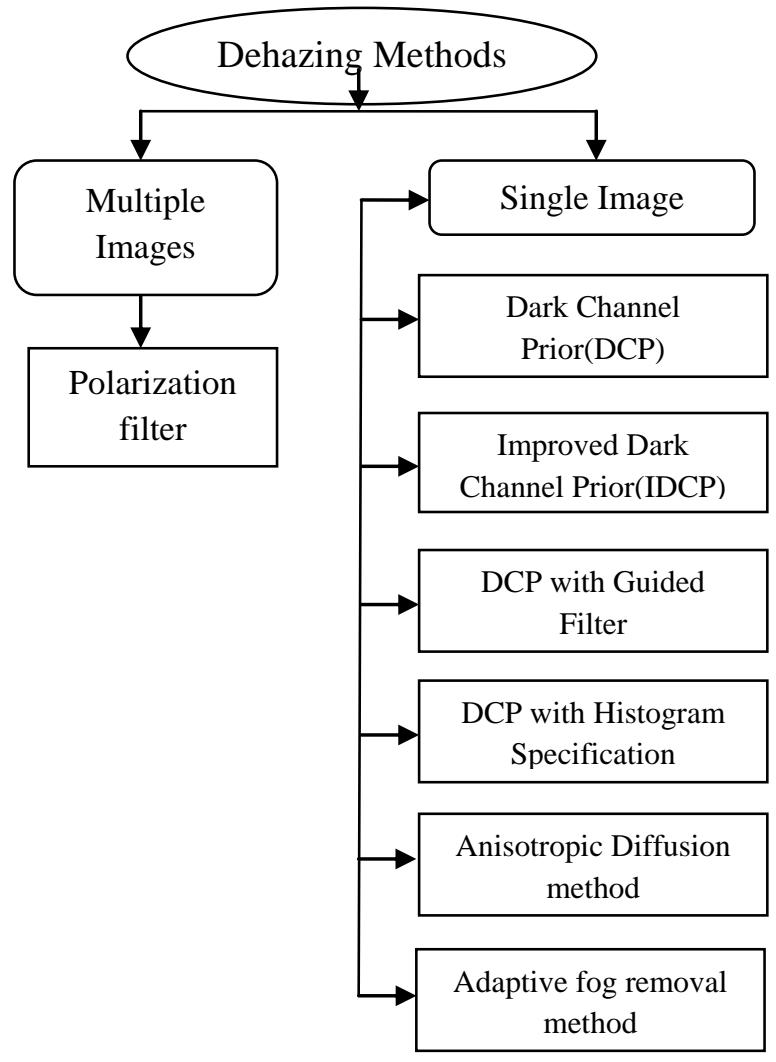

Fig 3.Different dehazing methods [6]

\section{DEHAZING METHODS}

In the bad weather conditions the atmosphere contains the fog. Due to this, it can significantly reduce the color and contrast of the images. For the fog removal different methods are shown below.

\subsection{Multiple Images method}

\subsubsection{Polarization Filter}

Fog can be removed by multiple image method by using polarization filter from the foggy images. In this method multiple input images of the same scene that has been taken during different bad weather conditions. The amount of light scattered due to atmospheric particles in the same direction of direct light arriving in the camera is termed as Airlight. For the use of polarization filters, assume that the Airlight is partially polarized. The polarization filter alone cannot remove the fog from images. In this method, input image is combination of two unknown components. The first one is the scene radiance in the absence of the fog and the other one is Airlight. This method doesn't need the weather conditions to change and it can be applied at any time.

\section{ADVANTAGES:}

- $\quad$ Fog is removed by the polarization filter.

- There is not Halo effects in the haze free image.

DISADVANTAGES:

- For this method multiple images with same scene and different weather conditions are require.

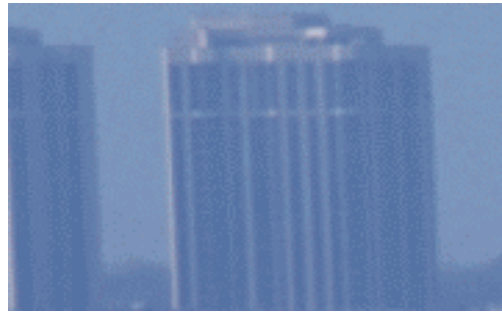

Fig 4. Hazy Image[24]

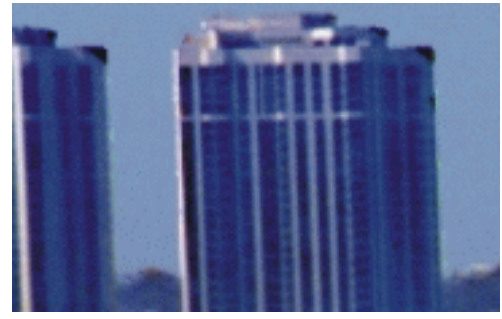

Fig 5. Dehazed Image [24]

\subsection{Single Image methods}

\subsubsection{Dark Channel Prior}

Dark channel prior is basically used for single image dehazing method. It is used to measure the statistics of the outdoor fog free image. In this method assume that some pixels are having very low intensity in any one of the color channel. But in this case, take only that region which does not cover the sky. These pixels are known as the dark pixels. These dark pixels are used to estimate the transmission map. After the transmission map estimation refined transmission map is estimated to remove some blocky effect. The aim of this technique is to restore fog free image. Main advantages of this method are in this method Single image is used for restoration of foggy image and Transmission map is estimated accurately.

\section{ADVANTAGES:}

- Single image required for haze removal.

- Transmission map is estimated accurately.

\section{DISADVANTAGES:}

- For Airlight estimate assumption is required that only $0.1 \%$ brightest pixels are taken.

- It produces some Halo effects on the resultant images.

- This method is invalid when scene object is similar to Airlight like car head lights, snowy ground, etc. 


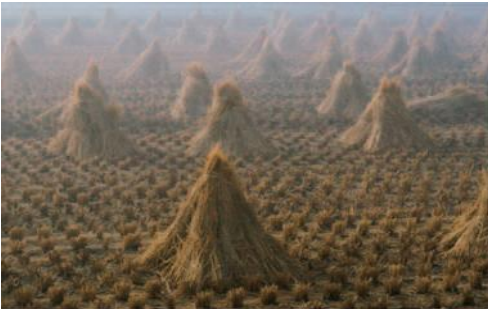

Fig 6. Hazy Image [18]

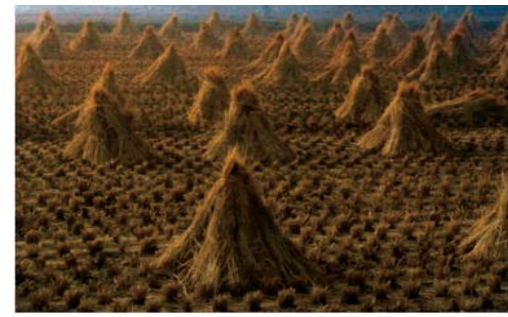

Fig 7. Dehazed Image [18]

\subsubsection{Improved Dark Channel Prior (IDCP)}

This method is improved version of Dark Channel Prior (DCP). Improvement is that soft-matting will be done using bilateral filter. The aim of applying bilateral filter is to smoothen the small scale textures of image. Improved Dark Channel prior is based on dark channel concept to estimate the atmospheric light and obtain better results. Most image dehazing methods only consider that use a hard threshold assumptions or user to estimate atmospheric light. The brightest pixels are sometimes the objects such as car lights or streetlights. So the wrong atmospheric light estimation may affect the dehazing results. It is resolves the problem of significant region which contains sky. Means in that sky regions also take as patches for obtain the atmospheric light. Results of the dark channel prior gives dim image after the haze removal. So, this method has greater efficiency, less execution time and improves the original algorithm.

\section{ADVANTAGES:}

- Estimation of air light is accurately.

- Complexity of soft matting for refined transmission is not requiring. So computation time is requiring less.

- Sky regions become bright and smoother.

\section{DISADVANTAGES}

- It produces Halo effects in some regions.

- Transmission map is not estimated accurately.

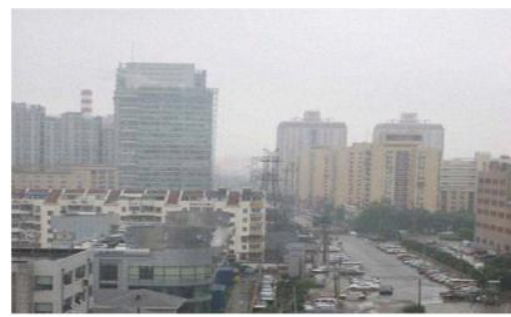

Fig 8. Hazy Image [17]

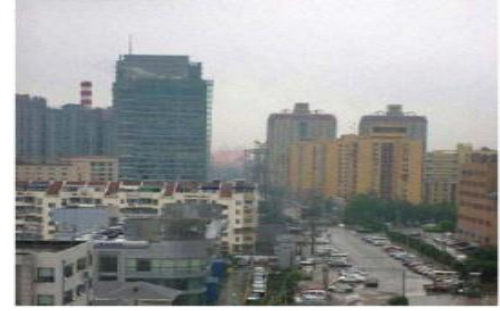

Fig 9. Dehazed Image [17]

\subsubsection{IDCP using Guided Filter}

Main disadvantage of DCP and IDCP method is that they produce halo effects (blocky effects) in the output image. So IDCP with guided filter method is introducing to overcome this disadvantage. Image dehazing will be done by combining dark channel prior and guided filter. Guided Filter is an edgepreserving smoothing operator. So it could remove halo artifacts effectively Dark channel is same as DCP method and atmospheric light is estimated based on the imaging law of very dense hazy regions. In that method transmission map is refined using guided filter. Refinement of transmission map is needed to remove the halo effects. Main advantages of this method are that estimation of refine transmission map is done accurately and it could get more accurate value of the atmospheric light. So due to this the haze-free image is not looking dim.

\section{ADVANTAGES:}

- In this method Halo effects is remove efficiently.

- Refined transmission is done by guided filter so it gives good result.

\section{DISADVANTAGES:}

- Estimation of Airlight is not done accurately.

- It can't able to improve the contrast.

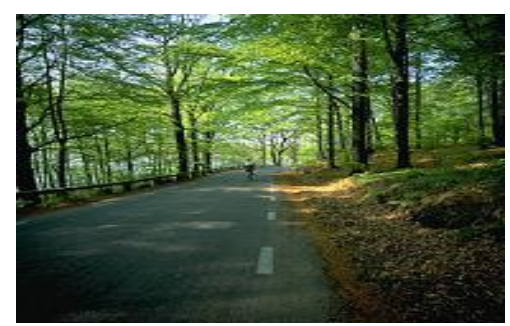

Fig 10. Hazy Image [6]

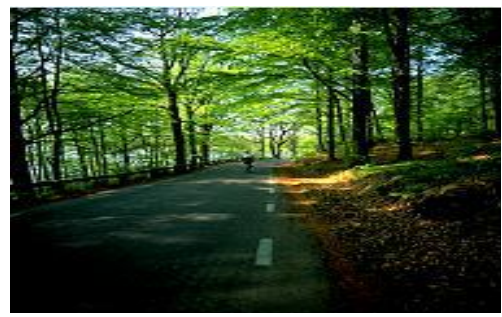

Fig 11. Dehazed Image [6]

\subsubsection{DCP with Histogram Specification}

DCP method is graceful and concise but there are some problems like thickness of haze, direct attenuation is not suitable for foreground. . So due to these reasons haze free 
image is looking dim. When removing the haze in the image with large background area and low contrast, DCP will not give good results To overcome these problems, DCP with histogram specification has been introduce to improve the contrast of the recovered image. This is resolved by rebuilding the histogram of image. For this method image with large background area and low contrast, and build the histogram of this kind of haze images together with their haze removal result. Atmospheric light and transmission map is estimated same as DCP. Histogram of haze removal image has a tendency of left-shifting and narrow, and some sharp points occur in the high intensity region. After that rebuild the histogram of haze removal image by expand the low intensity area of the histogram and eliminate the sharp point at the high intensity area. Benefit of Histogram specification is that change intensity of the interested area and other is invariant. This method gives much better result than DCP method.

\section{ADVANTAGES:}

- Haze is removed from the large background and low contrast images.

- Improved the contrast of the haze free image.

\section{DISADVANTAGES:}

- This method gives poor contrast image.

- Computation time is required more.

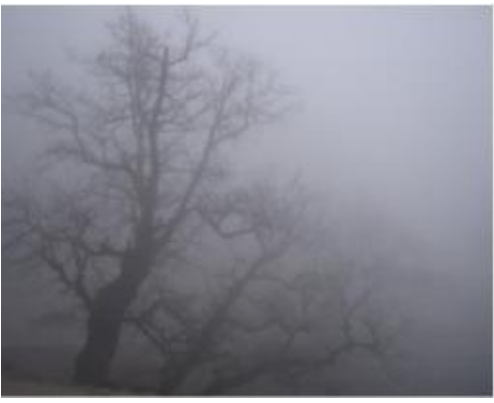

Fig 12. Hazy Image [17]

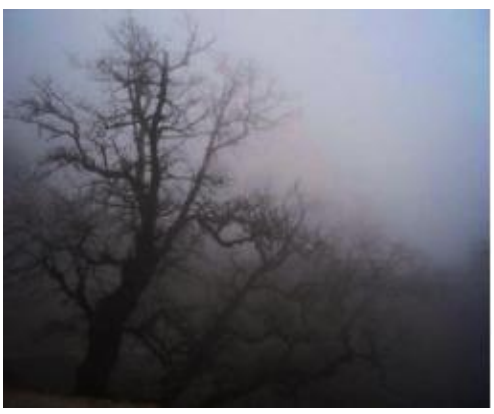

Fig 13. Dehazed Image [17]

\subsubsection{Anisotropic Diffusion Method}

Using this method improve the contrast of an image in HSI (hue, saturation and intensity) color plane without user interference. In this method anisotropic diffusion is used to refine the atmospheric light obtained using DCP method. This method is widely used in consumer electronics, tracking and navigation, and entertainment industries, etc. For the post processing histogram stretching will be done. Post processing is the contrast enhancement and it has done by various methods which are suitable for hazy image. HIS color model reduces the computation time. Main advantages of this method are estimation of the Airlight done accurately and improve the contrast of an image.

\section{ADVANTAGES:}

- Estimation of Airlight is done accurately.

- Contrast is enhanced in resultant image.

\section{DISADVANTAGES:}

- Transmission map is not estimated accurately.

- Visibility of the restored image is poor.

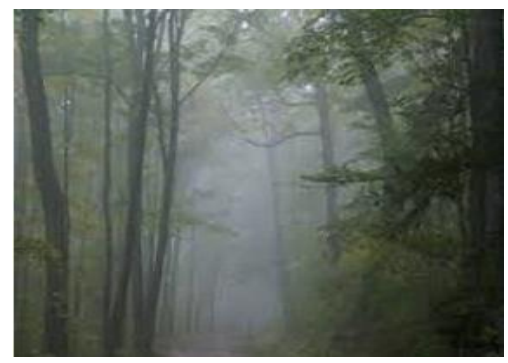

Fig 14. Hazy Image [5]

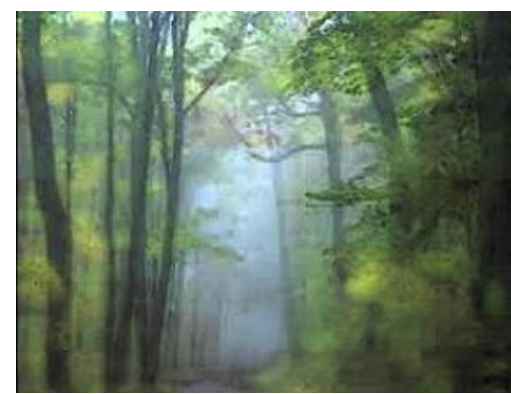

Fig 15. Dehazed Image [5]

\subsubsection{Adaptive fog removal Method}

It is hard to recover the original color and shape of an object in a hazy image. Single image based dehazing methods with additional depth information suffer from the color distortion problem. To solve this problem introducing the single image based algorithm using the luminance map. In this method HSV color model is used. Estimation of atmospheric light is using dark channel prior. This method takes highest intensity (V) value in the HSV color model for the estimation of atmospheric light. It is advantageous from other techniques in the sense that the other techniques take the brightest pixel among all color channels in the foggy image. After that luminance map is generated using the luminance of the image. Luminance map can be estimated using the average color of the pixels. The luminance map is generated from the $\mathrm{Y}$ channel of the image. So using atmospheric light and luminance map haze could be removed easily.

\section{ADVANTAGES:}

- Airlight is estimated accurately.

- Network performance for error and Peak Signal to Noise ratio is higher.

- It improves the image contrast.

DISADVANTAGES:

- Computation time is requiring more. 


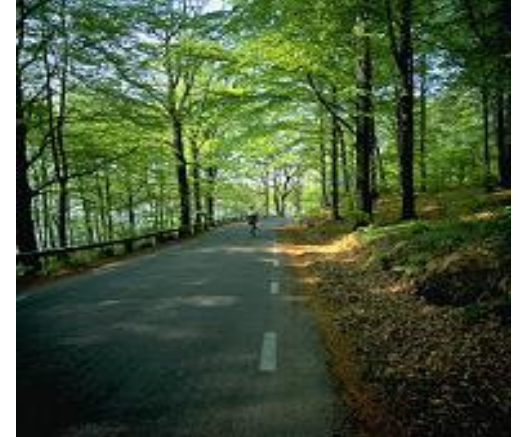

Fig 16. Hazy Image [6]

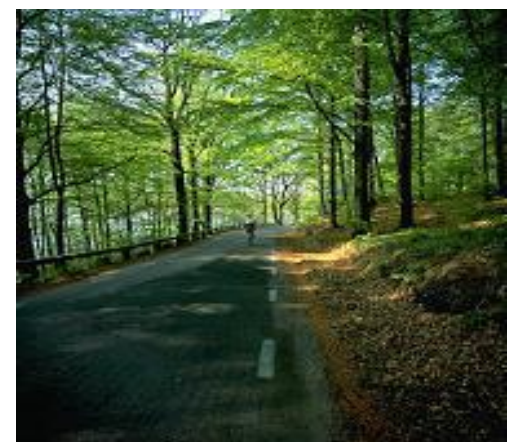

Fig 17. Dehazed Image [6]

\section{CONCLUSION}

This paper presents all simple and faster dehazing method in which the haze can be estimated from the captured hazy images. Haze removal methods have become more useful for many computer vision applications. All the dehazing methods useful for surveillance, intelligent vehicles, for remote sensing and under water imaging, etc. These methods are based on the partial estimation of atmospheric light. Above methods are not worked when the scene objects are inherently similar to the atmospheric light and no shadow is cast on them (such as the Snowy Ground). So in future implementing the best and fast defogging method to eliminate these problems. And also work for mist, rainy and snowy and camera mis-focused images.

\section{REFERENCES}

[1] Bingquan Huo and Fengling Yin, "Image Dehazing With Dark Channel Prior And Novel Estimation Model", International Journal of Multimedia and Ubiquitous Engineering Vol. 10, No. 3 (2015).

[2] Vinkey Sahu and Vinkey Sahu, "A Survey Paper On Single Image Dehazing", IJRITCC Volume: 3 Issue: 2 February 2015 .

[3] Dilraj Kaur and Pooja, "A Critical Study and comparative Analysis of Various Haze Removal Technique", International Journal of Computer Applications (0975 - 8887) Volume 121 - o.16, July 2015.

[4] Yishu Zhai, Dongjiang Ji, "Single Image Dehazing For Visibility Improvement", International Conference on Unmanned Aerial Vehicles in Geomatics, 30 Aug-02 Sep 2015.

[5] Pranjal Garg, Shailender Gupta, Bharat Bhushan and Prem Chand Vashist, "A Hybrid Defogging Technique Based On Anisotropic Diffusion And IDCP Using
Guided Filter", International Journal of Signal Processing, Image Processing and Pattern Recognition Vol.8, No.7 (2015).

[6] Atul Gujral, Aditi, Shailender Gupta and Bharat Bhushan, "A Comparison Of Various Defogging Techniques", International Journal of Signal Processing, Image Processing and Pattern Recognition Vol.7, No.3 (2014).

[7] Shih-Chia Huang, Bo-Hao Chen, and Wei-Jheng Wang, "Visibility Restoration Of Single Hazy Images Captured In Real-World Weather Conditions", IEEE Transactions on Circuits and Systems for Video Technology 2014.

[8] Harpoonamdeep Kaur, Dr. Rajiv Mahajan, "A Review on Various Visibility Restoration Techniques", International Journal of Advanced Research in Computer and Communication Engineering Vol. 3, Issue 5, May 2014.

[9] Gagandeep Singh, "Evaluation Of Various Digital Image Fog Removal Algorithms", International Journal of Advanced Research in Computer and Communication Engineering Vol. 3, Issue 7, July 2014.

[10] Shuai Yang, Qingsong Zhu, Jianjun Wang, Di Wu, and YaoqinXie, "An Improved Single Image Haze Removal Algorithm Based On Dark Channel Prior And Histogram Specification", 2013.

[11] Ashok Shrivastava, Er. Rekha Kumari, "Review on Single Image Fog Removal", International Journal of Advanced Research in Computer Science and Software Engineering Volume 3, Issue 8, August 2013.

[12] A. K. Tripathi and S. Mukhopadhay, "Single Image Fog Removal using Anisotropic Diffusion", IET Image Processing, vol. 6, no. 7, 2012.

[13] Tripathi, A. K., and S. Mukhopadhyay. "Single image fog removal using bilateral filter." Signal Processing, Computing and Control (ISPCC), 2012 IEEE International Conference on. IEEE, 2012

[14] I. Yoon, J. Jeon, J. Lee, and J. Paik, "Spatially Adaptive Image Defogging Using Edge Analysis And GradientBased Tone Mapping", Proc. IEEE Int. Conf. Consumer Electronics, January 2011.

[15] Jiahao Pang, Oscar C. Au and Zheng Guo, "Improved Single Image Dehazing Using Guided Filter", APSIPA ASC 2011.

[16] Kaiming He, Jian Sun, and Xiaoou Tang, "Single Image Haze Removal Using Dark Channel Prior", IEEE Transactions on Pattern Analysis and Machine Intelligence, Vol. 33, No. 12, December 2011.

[17] Yan Wang and Bo Wu, "Improved Single Image Dehazing using Dark Channel Prior", IEEE 2010.

[18] K. He, J. Sun and X. Tang, "Single Image Haze Removal Using Dark Channel Prior", IEEE Int. Conf. on Computer Vision and Pattern reorganization, 2009.

[19] R. T. Tan, "Visibility in bad weather from a single image", in IEEE Conf. on Computer Vision and Pattern Recognition, 2008.

[20] K. Garg, S. K. Nayar, "Vision and rain", Int. J. Comput.Vis., 2007. 
[21] A. S. Narasimhan and S. Nayar, "Contrast restoration of weather degraded images," IEEE Trans. Pattern Analysis and Machine Intelligence, vol. 25, no. 6, June 2003.

[22] Y.Y. Schechner, S.G. Narasimhan, and S.K. Nayar, "Polarization Based Vision Through Haze," Proc. Applied Optics, special issue: light and color in the open air., vol. 42, no. 3, Jan. 2003.
[23] Srinivasa G. Narasimhan And Shree K. Nayar, "Vision and the Atmosphere", International Journal of Computer Vision 48(3), 2002.

[24] Yoav Y. Schechner, Srinivasa G. Narasimhan and Shree K. Nayar, "Instant Dehazing Of Images Using Polarization", Proc. Computer Vision \& Pattern Recognition Vol. 1(2001). 